\title{
Review
}

\section{Structural studies of functional nucleosome complexes with transacting factors}

\author{
By Hitoshi KurumizaKA ${ }^{* 1, \dagger}$ \\ (Edited by Shigekazu NAGATA, M.J.A.)
}

\begin{abstract}
In eukaryotic cells, the genomic DNA is hierarchically organized into chromatin. Chromatin structures and dynamics influence all nuclear functions that are guided by DNA, and thus regulate gene expression. Chromatin structure aberrations cause various health issues, such as cancer, lifestyle-related diseases, mental disorders, infertility, congenital diseases, and infectious diseases. Many studies have unveiled the fundamental features and the heterogeneity of the nucleosome, which is the basic repeating unit of chromatin. The nucleosome is the highly conserved primary chromatin architecture in eukaryotes, but it also has structural versatility. Therefore, analyses of these primary chromatin structures will clarify the higher-order chromatin architecture. This review focuses on structural and functional studies of nucleosomes, based on our research accomplishments.
\end{abstract}

Keywords: chromatin, nucleosome, histones, epigenetics, transcription, structure

\section{Introduction}

Living organisms use genomic DNA as the carrier of their genetic information. The length of human genomic DNA is estimated to be about 2 meters, and yet it is properly packaged in the nucleus, with a diameter of less than 10 micrometers. Genomic DNA is folded as a nucleoprotein complex called chromatin, which itself is organized by nucleosomes.

The history of chromatin research begins with Walther Flemming, who coined the term "chromatin" (from the Greek kroma, meaning color) in the 1880s to identify a stainable substance found in the cell nucleus. ${ }^{1)}$ Almost a century later, initial structural

*1 Laboratory of Chromatin Structure and Function, Institute for Quantitative Biosciences, The University of Tokyo, Tokyo, Japan.

$\dagger$ Correspondence should be addressed: H. Kurumizaka, Laboratory of Chromatin Structure and Function, Institute for Quantitative Biosciences, The University of Tokyo, 1-1-1 Yayoi, Bunkyo-ku, Tokyo 113-0032, Japan (e-mail: kurumizaka@iqb. u-tokyo.ac.jp).

Non-standard abbreviation list: cGAS: cyclic GMP-AMP synthase; Cryo-EM: cryo-electron microscopy; H3K9me3: lysine 9trimethylated H3; H3K27me3: lysine 27-trimethylated H3; HP1: heterochromatin protein 1; OLDN: overlapping dinucleosome; PRC2: polycomb repressive complex 2; RNAPII: RNA polymerase II; SHL: superhelical location; TF: transcription factor. images of chromatin were published in 1974, in a report where the authors used electron microscopy to observe spherical particles tethered by linker DNAs, which they called " $\nu$-bodies". ${ }^{2)}$ Subsequently, the term "nucleosome" replaced the name " $\nu$-body". In 1977, a broad outline of the nucleosome core particle conformation was reported, based on X-ray diffraction and electron microscopy analyses, revealing its disk-like shape with a diameter of $110 \AA$ and a height of $57 \AA .{ }^{5)}$ In 1984, the structure of the nucleosome core particle, in which the DNA forms a left-handed superhelix, was solved at $7 \AA$ resolution by X-ray crystallography. ${ }^{6)}$ In 1997, the atomic resolution structure of the nucleosome core particle was determined at $2.8 \AA$ resolution, revealing the details of the nucleosome structure, including histone-histone and histone-DNA interactions. $\left.{ }^{7}\right)$ Additional structural details were elucidated based on the higher resolution structure at $1.9 \AA$ resolution, obtained by X-ray crystallography. ${ }^{8), 9)}$

The nucleosome core particle, a $10 \mathrm{~nm}$ spherical unit, comprises two molecules each of the four core histones H2A, H2B, H3, and H4 (Fig. 1A), which assemble as a histone octamer tightly wrapped by $\sim 147$ base pairs (bp) of DNA in a left-handed superhelix (Fig. 1B). The histone octamer is composed of two H3-H4 heterodimers and two H2A-H2B 
A

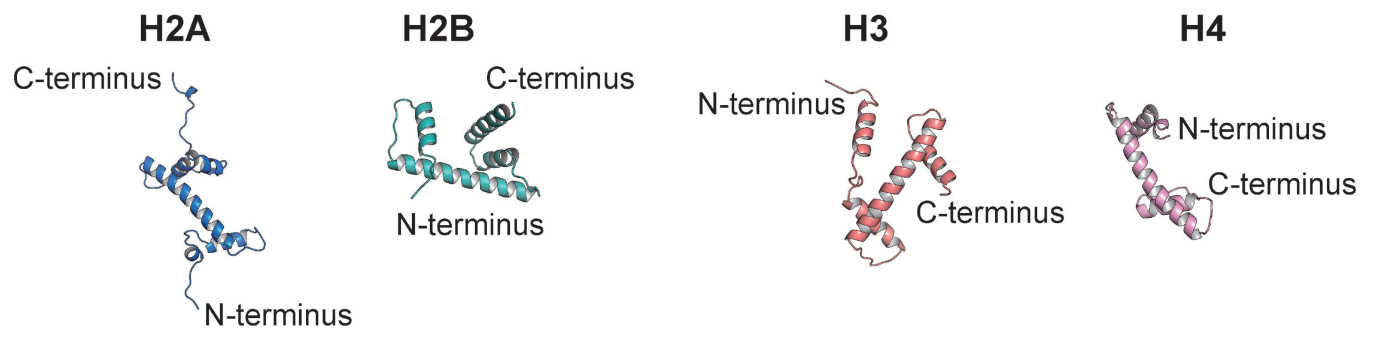

B
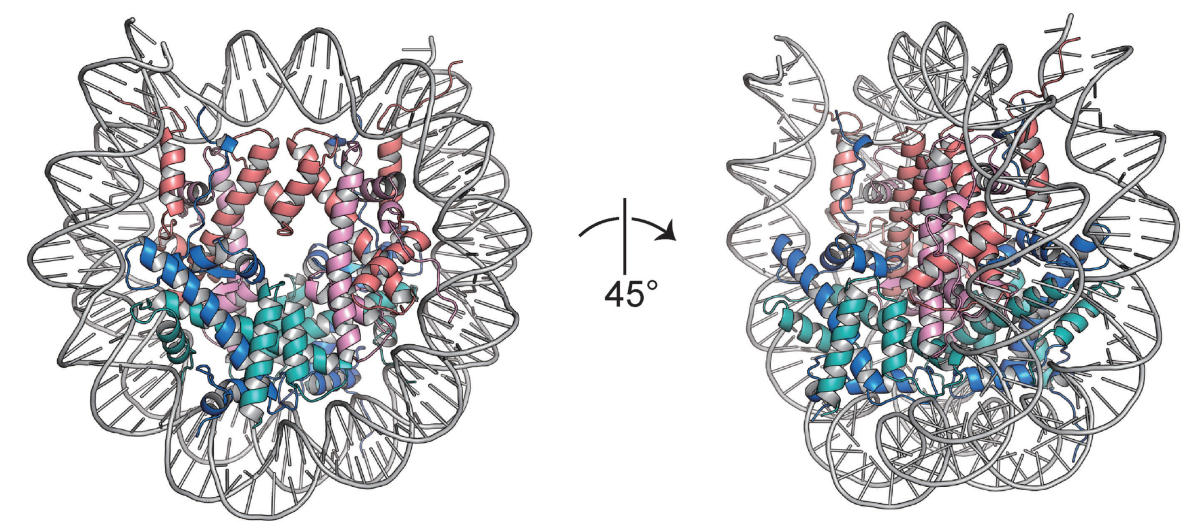

Fig. 1. Structure of the nucleosome. A. Histones H2A, H2B, H3, and H4 associate to form a nucleosome. Structural depiction of the nucleosomal histones H2A, H2B, H3, and H4, colored marine, turquoise, salmon pink, and pink, respectively. B. Crystal structure of the human canonical nucleosome core particle. The figure presents the human canonical nucleosome structure with the DNA, colored gray, wrapped around the histone octamer (left panel). The nucleosome structure is rotated 45 degrees clockwise around the axis (right panel).

heterodimers. This essential nucleosome architecture is rigorously conserved in eukaryotic cells. ${ }^{10)}$ The nucleosomes are linked together by a linker DNA containing target sites for various proteins and regulatory factors, and form the "beads-on-a-string" architecture observed by Olins and Olins in $1974 .^{2)}$ The dynamic interactions between DNA and histones in the nucleosome are fundamental to gene regulation mechanisms mediated by chromatin, controlling all processes involving genomic DNA, such as gene transcription, DNA repair, recombination, and replication.

Sequence variations in genomic DNA are the driving force behind evolution and the acquisition of diversity in living organisms. At the same time, changes in genomic DNA sequences can be the source of serious diseases, such as cancer and neurodegenerative disorders. On the other hand, disorders caused by the genomic organization occurring in a DNA sequence-independent manner have recently been identified. These disorders are not the consequences of alterations in the genetic code of the DNA sequence, but instead are caused by the mechanism known as "epigenetics". Epigenetics comprises heritable changes in the structure and organization of chromatin, and it plays a central role in the development of tissues and organs through cell differentiation. The genomic state that ensures the epigenetic control of genomic DNA is called the "epigenome", which is composed of chromatin bearing an array of modifications and factors, such as DNA methylation, post-translational modifications of histones, histone variants, nucleosome binding factors, nucleosome remodeling factors, and non-coding RNAs. These major epigenomic factors, by acting alone or in combination, result in the dynamic epigenetic regulation of genomic DNA by diversifying the chromatin structure and dynamics.

Over the past two decades, discussions of the structural elements of chromatin have mainly been based on the canonical nucleosome structure. Despite extensive efforts, the chromatin structure-based regulatory mechanisms of genomic DNA remain unclear. Therefore, deciphering the mechanism by 
which epigenetic factors regulate the chromatin state will not only contribute to a better understanding of the functional regulation of genomic DNA, such as gene expression, replication, repair, and recombination, but also provide important information for the establishment of new therapeutic and preventive methods for diseases caused by epigenetic aberrations.

This review summarizes structural studies of the nucleosome based on our research conducted over the past two decades, which has been dedicated to the clarification of the molecular mechanisms by which eukaryotic organisms use the epigenetic information encoded in higher-order chromatin structures.

\section{In vitro reconstitution of nucleosomes}

The basic chromatin architecture is established during early embryogenesis by histone assembly. To understand the mechanism of histone incorporation into chromatin, we analyzed the process by which histones $\mathrm{H} 3$ and $\mathrm{H} 4$ are incorporated into nascent chromatin during the cleavage stages of Xenopus laevis embryos. In this study, mRNAs encoding mutant $\mathrm{H} 3$ or $\mathrm{H} 4$ molecules were microinjected into the nuclei of Xenopus embryos to study the mitosisdependent incorporation of the mutant histones into newly formed chromatin. ${ }^{11)}$ Although this method allows to identify the amino acid regions of histones important for nucleosome formation in vivo, analyses of the effects of amino acid differences found in histone variants and mutants on nucleosome structure and function proved to be difficult. Therefore, we established a new strategy in which nucleosomes are reconstituted in vitro with histones produced as recombinant proteins. ${ }^{12)}$ We successfully purified histones H3, H3 mutants, and $\mathrm{H} 4$ as recombinant proteins, and determined that the H3 mutations leading to a SWI/SNF-independent (Sin) phenotype in yeast destabilize nucleosomes in vitro. ${ }^{12)}$ Genetic experiments in yeast demonstrated that Sin mutations promote transcription in genes requiring nucleosome remodeling factors, in the absence of the nucleosome remodeling activity. ${ }^{13)-15)}$ Wechser et al. also reported that Sin mutations of $\mathrm{H} 4$ enhance the accessibility of the nucleosomal DNA. ${ }^{16)}$ Crystal structures of the nucleosome core particles containing Sin mutations revealed that these mutations alter the histone-DNA interactions in the nucleosome. ${ }^{17)}$ These results explained how nucleosome destabilization by Sin mutations on $\mathrm{H} 3$ and $\mathrm{H} 4$ relieves the transcription barrier by the nucleosome, which is usually accomplished by nucleosome remodeling factors.

\section{Chromatin structure of centromeres}

During each cell division, the sister chromatids of the same chromosome are attached to microtubule fibers that pull the chromatids to opposite poles in daughter cells. The centromere region of the chromosome provides sites for the formation of the kinetochore, a proteinaceous structure where microtubules are anchored. ${ }^{18)-21)}$ The centromeric chromatin structure is a fundamental architecture that mediates chromosome segregation and may be conserved among eukaryotes. The centromere formation position and the resulting chromatin structure are epigenetically maintained through cell division and passed on to the next generation.

CENP-B is one of the protein components of centromeric chromatin. ${ }^{22), 23)}$ By forming a dimeric structure, CENP-B recognizes and binds specifically to a target DNA sequence called the CENP-B box, located in the centromeric chromatin. ${ }^{24)-28)} \mathrm{We}$ solved the crystal structures of the CENP-B Nterminal DNA-binding domain complexed with the CENP-B box DNA ${ }^{29)}$ and the CENP-B dimerization domain. ${ }^{30)}$ To analyze nucleosome binding factors such as CENP-B, the previously established nucleosome reconstitution system using recombinant histones seemed to be effective. Therefore, we developed a system for the expression and purification of recombinant human histones in Escherichia coli cells. ${ }^{31), 32)}$

To enhance our knowledge of the structural architecture of the centromeric nucleosome, we focused on the centromere-specific histone $\mathrm{H} 3$ variant CENP-A, which is specifically assembled in nucleosomes localized in the centromeric region. Although the underlying mechanism remains unclear, it is well known that centromere inheritance is dictated by the architecture of the centromeric nucleosome containing CENP-A. Human CENP-A was successfully obtained as a recombinant protein, and the nucleosome comprising CENP-A was demonstrated to form a nucleosome-like structure with the other histones, $\mathrm{H} 2 \mathrm{~A}, \mathrm{H} 2 \mathrm{~B}$, and $\mathrm{H} 4$. Using the reconstituted human centromeric nucleosomes, we demonstrated that CENP-B binds efficiently to nucleosomal DNA in the presence of CENP-A, even when the CENP-B box is wrapped within the nucleosome. ${ }^{33), 34)}$

CENP-A has attracted attention as an epigenetic factor that defines the centromere region of chromosomes. The centromeric nucleosome is considered to adopt a unique structure dedicated to kinetochore formation. Although several models of the CENP-A nucleosome were proposed and actively 

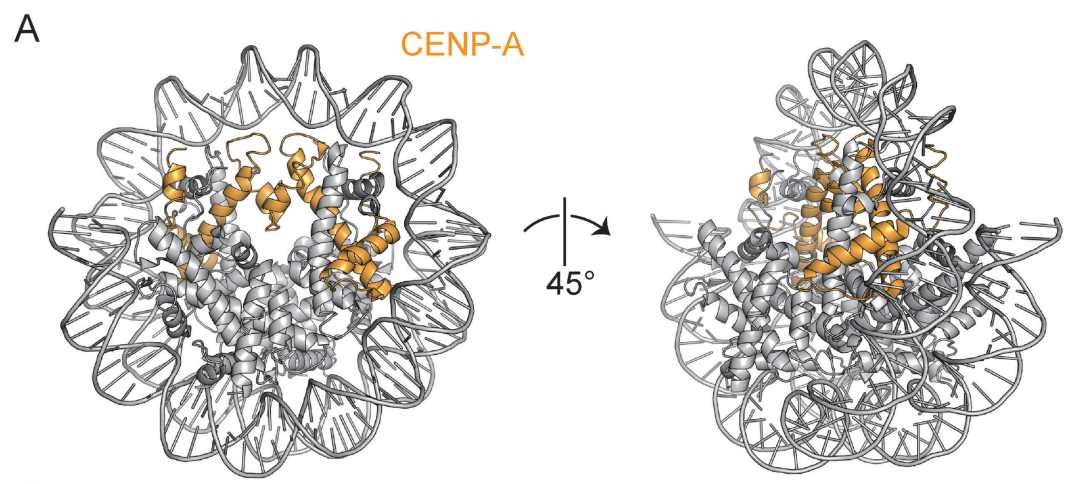

B

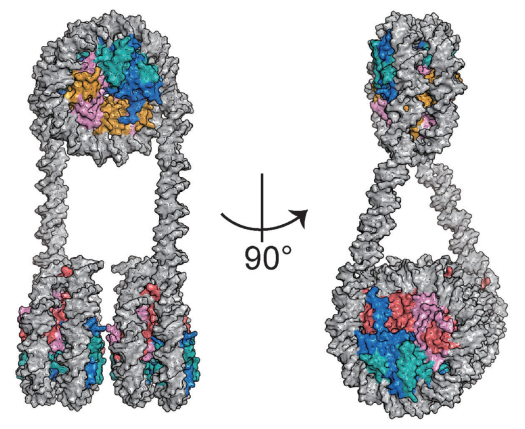

H3.1-CENP-A-H3.1 tri-nucleosome

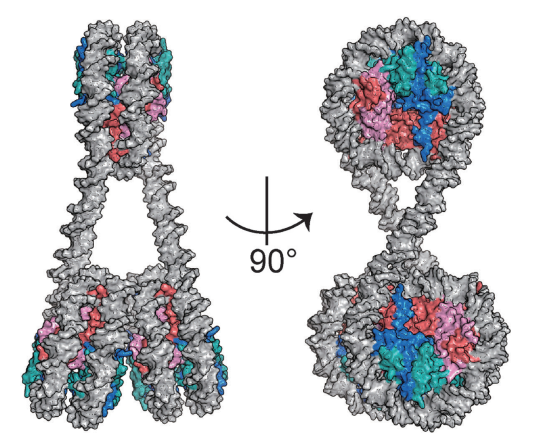

H3.1-H3.1-H3.1 tri-nucleosome

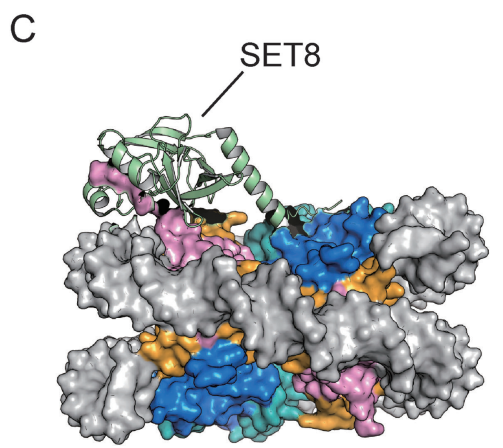

Fig. 2. Centromeric chromatin architecture. A. Crystal structure of the CENP-A nucleosome. The figure presents the nucleosome core particle structure containing centromeric CENP-A, colored gold. DNA is colored gray. The right panel shows the CENP-A nucleosome rotated 45 degrees clockwise around the axis. B. Cryo-EM structures of trinucleosomes containing CENP-A or canonical H3.1. Front and right-angle views of the tri-nucleosomes containing CENP-A or H3.1 are presented. The four histones H2A, H2B, H3.1 and H4 are colored marine, turquoise, salmon pink, and pink, respectively. The DNA is colored gray. C. Interaction of the methyltransferase SET8 with the H4 N-terminal tail of the nucleosome and the CENP-A nucleosome, analyzed using cryo-EM. Close-up view of the H4 N-terminal tail of the CENP-A nucleosome bound to the SET domain, colored mint. Histones H2A, H2B, CENP-A, H4, and DNA are colored marine, turquoise, orange, pink, and gray, respectively.

discussed, the three-dimensional structure of nucleosomes containing CENP-A had remained unknown. ${ }^{35)}$ Therefore, the determination of the enigmatic structure of the centromere-specific CENP-A nucleosome was eagerly awaited. We reconstituted the human CENP-A nucleosome with a centromeric
DNA fragment and successfully determined its crystal structure $^{36)}$ (Fig. 2A). The overall structure of the CENP-A nucleosome was quite similar to the structures of nucleosomes containing other histone H3 variants. ${ }^{37), 38)}$ Notably, the DNA ends were flexible, a property that may play an important role 
in forming the centromeric chromatin architecture facilitating the assembly of centromeric proteins. Consistently, the DNA end flexibility and structural diversity in the CENP-A nucleosomes have been confirmed by cryo-electron microscopy (cryo-EM) analyses. $^{39), 40)}$ However, the extent to which the flexible DNA ends of the CENP-A nucleosome participate in the establishment of the higher-order structure of centromeric chromatin had remained unclear.

To address this question, we established a method to reconstitute tri-nucleosomes mimicking a centromeric nucleosome arrangement, in which the CENP-A nucleosome is flanked by two H3 nucleosomes. The cryo-EM single-particle analysis revealed that the tri-nucleosomes containing CENP-A form a higher-order structure that is easily accessible by centromeric proteins in chromatin, as compared with tri-nucleosomes containing only $\mathrm{H} 3$ nucleosomes ${ }^{41)}$ (Fig. 2B). Notably, active centromere formation with a CENP-A mutant that can be localized in the centromeres, but is defective in DNA end flexibility, was substantially impaired. ${ }^{39)}$ These results provided important insights into the functions of histone variants in the proper formation of the higher-order architecture of chromatin.

Our analyses also revealed that, in the nucleosome, CENP-A fixes the orientation of the Nterminal tail of the cognate $\mathrm{H} 4$ in the outward configuration. ${ }^{42)}$ In the previous crystal structures of canonical human nucleosomes, the inward configuration of the $\mathrm{H} 4 \mathrm{~N}$-terminal tail was predominantly observed. ${ }^{37), 38)}$ Cryo-EM analyses of CENP-A and H3.1 nucleosomes complexed with the monomethyltransferase SET8 revealed that the outward configuration of the $\mathrm{H} 4 \mathrm{~N}$-terminal tail is suitable for SET8 binding to the nucleosome ${ }^{43)}$ (Fig. 2C). Consistently, SET8 monomethylates the CENP-A nucleosome more efficiently than the canonical nucleosome. ${ }^{42)}$ These findings explained how CENP-A enhances the monomethylation of the $\mathrm{H} 4$ lysine 20 residue in the CENP-A nucleosome. ${ }^{44)}$

In addition to its importance in centromeric chromatin formation, CENP-A is overexpressed in particularly aggressive cancer cells and forms the heterotypic nucleosome containing one CENP-A molecule together with one H3.3 molecule at noncentromeric chromatin regions. ${ }^{45}$ We successfully reconstituted the CENP-A/H3.3 heterotypic nucleosome, and determined its crystal structure. In the structure, CENP-A and H3.3 retained their structural characteristics, and the DNA end was flexible only on the CENP-A side. ${ }^{46)}$ How these heterotypic nucleosomes facilitate cancer progression will be an important future issue to resolve.

\section{Testis-specific histone variants}

Dramatic chromatin structure reorganization is induced during spermatogenesis. Several histone variants are specifically expressed in the testis and induce structural changes in chromatin. H3T was identified as the testis-specific histone H3 variant in mammals and is regarded as a critical histone variant that plays an important role in chromatin reorganization required for spermatogenesis.

We purified human H3T as a recombinant protein, and found that H3T is apparently incorporated into the nucleosome by a specific pathway with the histone chaperone Nap2. ${ }^{47}$ The three-dimensional structure of the H3T-containing nucleosome core particle was solved, and its physical and biochemical characteristics were determined. ${ }^{48)}$ The H3T nucleosome is unstable, and the amino acid residues responsible for the instability have been identified. ${ }^{48)}$ A mouse H3T ortholog (termed H3t) was detected by in silico hybridization screening. $\left.{ }^{49}\right)$ Structural, biochemical, and knockout analyses revealed that $\mathrm{H} 3 \mathrm{t}$ is essential for spermatogenesis, although the mouse H3t nucleosome exhibited somewhat different physical and biochemical characteristics from those of the human H3T nucleosome. ${ }^{50)}$ These physical and structural properties of H3Tcontaining nucleosomes may provide important information for understanding the basis of chromatin reorganization during spermatogenesis. Studies of other testis-specific histone variants, such as human H3.5, ${ }^{51)}$ human TSH2B, ${ }^{52)}$ mouse and human TH2A and TH2B, ${ }^{53)-56)}$ and mouse H2A.L. $2^{57}$ ) have been reported. It is intriguing to elucidate the functions of these diverse nucleosomes in the chromatin reorganization during spermatogenesis.

\section{Nucleosomes in heterochromatin}

Heterochromatin represents chromosomal regions where DNA is densely condensed. By ensuring a condensed high-order chromatin conformation, heterochromatin suppresses genomic DNA activities, such as transcription, replication, recombination, and repair. Therefore, heterochromatin is an important element that plays a fundamental role in epigenetics. Two classes of heterochromatin have been identified: constitutive heterochromatin and facultative heterochromatin. Constitutive heterochromatin is marked by the lysine 9-trimethylated H3 (H3K9me3) mod- 
A

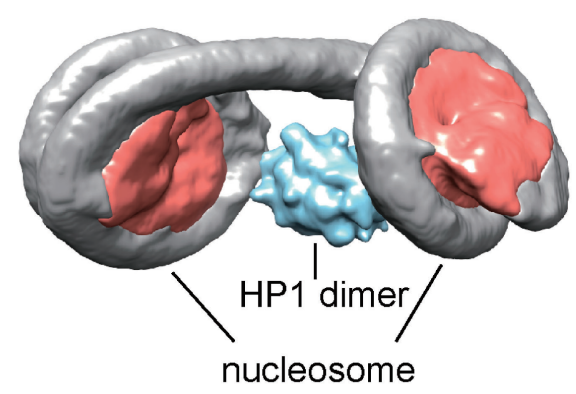

B

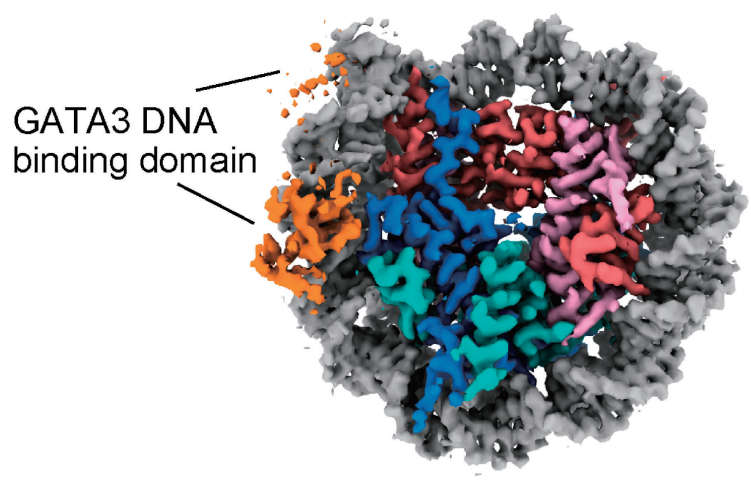

C

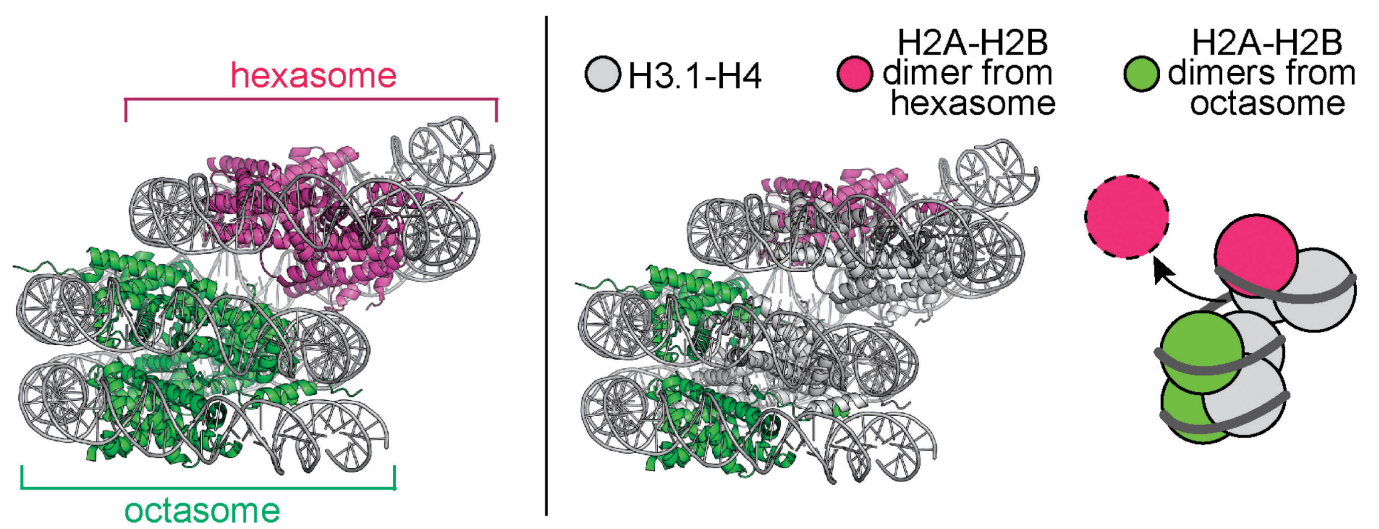

Fig. 3. Diversification of the primary chromatin architecture. A. Cryo-EM structure of the HP1 $\alpha$-dinucleosome complex. The two nucleosomes and the HP1 $\alpha$ molecule are colored salmon pink and light blue, respectively. The DNA is colored gray. B. Cryo-EM structure of the DNA binding domain of GATA3 complexed with the nucleosome core particle. The DNA binding domain of the pioneer transcription factor GATA3, colored orange, is bound to the nucleosome. Histones H2A, H2B, H3.1, H4, and DNA are colored marine, turquoise, salmon pink, pink, and gray, respectively. C. Crystal structure of the overlapping dinucleosome. The octasome and hexasome moieties are shown in light green and magenta, respectively. The DNA is colored gray (left panel). The right panel represents the $\mathrm{H} 2 \mathrm{~A}-\mathrm{H} 2 \mathrm{~B}$ dimer content in the hexasome and octasome units. The $\mathrm{H} 2 \mathrm{~A}-\mathrm{H} 2 \mathrm{~B}$ dimers contained in the hexasome and octasome units are presented in magenta and light green, respectively. The $\mathrm{H} 3.1-\mathrm{H} 4$ content in the two units is presented in gray. The H2A-H2B dimer colored in pink with broken line corresponds to the dimer that has detached from the octasome.

ification and heterochromatin protein 1 (HP1), which specifically binds to chromatin containing H3K9me3. In comparison, facultative heterochromatin usually contains the lysine 27-trimethylated H3 (H3K27me3) modification, which is catalyzed by polycomb repressive complex 2 (PRC2).

The mechanism by which HP1 binds to the H3K9me3 nucleosome had remained enigmatic. We first purified a histone H3 containing a trimethyllysine 9 analog, which mimics H3K9me3, and then obtained complexes of HP1 with the dinucleosome containing H3K9me3. The cryo-EM structures of human HP1 subtypes (HP1 $\alpha$, HP1 $\beta$, and $\mathrm{HP} 1 \gamma)$ in complexes with the H3K9me3 dinucleosome were elucidated by single-particle analysis. ${ }^{58)}$ In the complexes, an HP1 homodimer binds to each nucleosome of the dinucleosome, probably by binding H3K9me3, and bridges two nucleosomes (Fig. 3A). Notably, nucleosome bridging was also observed in the cryoEM structure of facultative heterochromatin complexes with PRC2. ${ }^{59)}$ These findings revealed the basic structural element of heterochromatin, and may provide important insights toward clarifying the common mechanism of gene inactivation in heterochromatin regions.

\section{Interactions of pioneer transcription factors with nucleosomes}

In the genome, transcription factors (TFs) recognize and bind their target DNA sequences to regulate gene transcription. The presence of nucleosomes generally restricts the access of TFs to their 
target DNA sequences. However, it has been reported that certain TFs, called pioneer TFs, bind to their target DNA sequences, even when they are wrapped within nucleosomes. ${ }^{60}$ ) Pioneer TFs thus cause conformational changes in chromatin that allow downstream factors to bind to their exposed target DNA sequences. Therefore, pioneer TFs are crucial elements involved in cell differentiation. However, the mechanism by which pioneer TFs recognize and bind to their target DNA sequences in nucleosomes had remained elusive, because of a lack of structural knowledge.

Our research group focused on the pioneer TF GATA3, involved in the establishment of new cell fates, including mammary epithelial cell differentiation by induction of chromatin remodeling, ${ }^{61)}$ and determined the cryo-EM structure of the nucleosome core particle complexed with the GATA3 DNA binding domains ${ }^{62)}$ (Fig. 3B). Together with in vitro biochemical and genomic analyses, we found that the positions of the target DNA sequences on the nucleosome are important for GATA3 to recognize and bind to the nucleosome. In fact, GATA3 stably binds to the nucleosome by effectively using its two DNA-binding motifs without obvious DNA unwrapping, when its target DNA sequence $5^{\prime}-\mathrm{GAT}-3^{\prime}$ is located near the nucleosomal edge and properly aligned in the same direction by six base-pair spacing. In contrast, the cryo-EM structures of nucleosomes complexed with other pioneer TFs, such as Sox2, Oct4, and Sox11, revealed that they bind to their target DNA sequences by partially unwrapping the DNA from the histone surface. ${ }^{63), 64)}$ Studies on pioneer TFs are providing important information toward clarifying the molecular mechanism by which these fundamental TFs bind their DNA targets within the nucleosome.

\section{Nucleosomes around transcription start sites}

Nucleosome formation inhibits the binding of DNA-binding proteins, such as TFs. When a nucleosome is located near the transcription start site, it is shifted along the DNA by nucleosome remodeling factors. This step allows the assembly of TFs, including RNA polymerase II, on the transcription start site, and thus the gene can be transcribed. During this process, the nucleosomes may collide with neighboring nucleosomes. ${ }^{65), 66)}$ To reveal the structure after nucleosome collision, we reconstituted the "overlapping dinucleosome (OLDN)", in which two nucleosomes have collided, and determined its crystal structure. ${ }^{67)}$ The OLDN structure consists of two units: one normal-type histone octamer and one hexamer lacking an H2A-H2B dimer, with about $250 \mathrm{bp}$ of DNA wrapped around the histone core in three turns (Fig. 3C). A molecular dynamics simulation coupled with small angle X-ray and neutron scattering revealed the dynamic characteristics of the OLDN in solution. ${ }^{68)}$ The results obtained from genomics, in conjunction with structural and biochemical analyses, also suggested that OLDNs accumulate just downstream of the transcription start sites in human cells. ${ }^{67)}$ Thus OLDNs may represent a novel chromatin unit that regulates transcription initiation by nucleosome repositioning during the chromatin remodeling process.

\section{Transcription elongation of nucleosomal DNA by RNA polymerase II}

In eukaryotes, RNA polymerase II (RNAPII) is the enzyme responsible for the transcription of protein-coding genes and non-coding RNAs. However, the genomic DNA that RNA polymerases must transcribe is tightly bound to the histone core in the nucleosome and thus lacks easily accessible sites for polymerase binding. Therefore, in the process of transcription on the nucleosome, RNA polymerases must disrupt the robust contacts between histones and DNA during transcript elongation. Although many structural studies on the mechanism of transcription by RNAPII on DNA have been performed, the mechanism by which RNAPII transcribes the nucleosomal DNA had remained unclear and thus was eagerly awaited. To address this fundamental question, we established a nucleosome transcription system with RNAPII, and pursued the single-particle analysis using cryo-EM. We successfully captured snapshot structures of RNAPII-nucleosome complexes at each step of nucleosomal DNA transcription $^{69)}$ (Fig. 4A). The RNAPII-nucleosome structures revealed that RNAPII performs transcription elongation reactions by tearing the nucleosomal DNA from the histone surface in a stepwise manner, while preserving the histone octamer. Notably, we also found that RNAPII substantially pauses near the entry and the center of the nucleosome, during the transcription elongation reaction. These partially DNA-peeled nucleosomes within the paused complexes may be the targets of histone chaperones, nucleosome remodelers, and modification enzymes.

Various transcription elongation factors escort RNAPII to promote efficient transcription through the nucleosome. However, the mechanism by which 
A

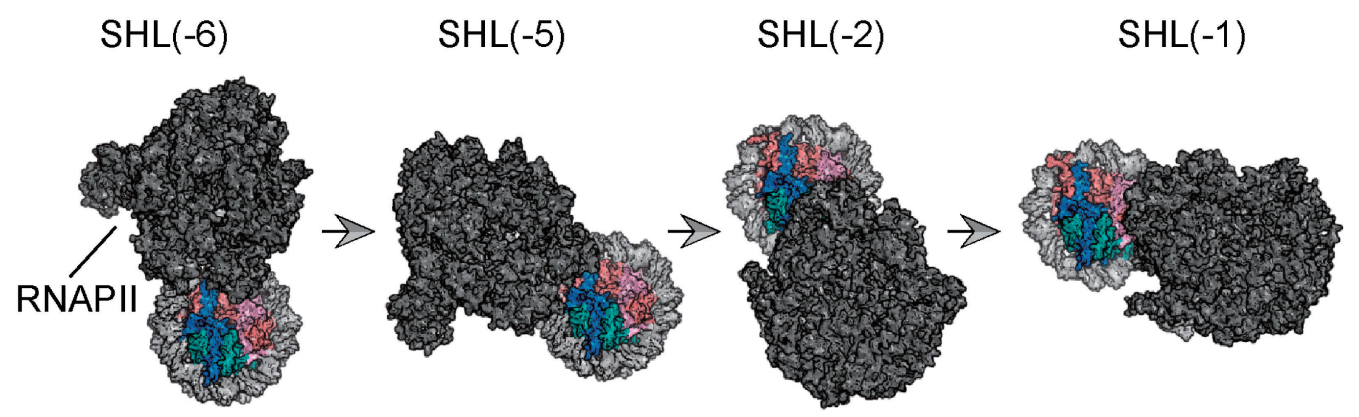

B

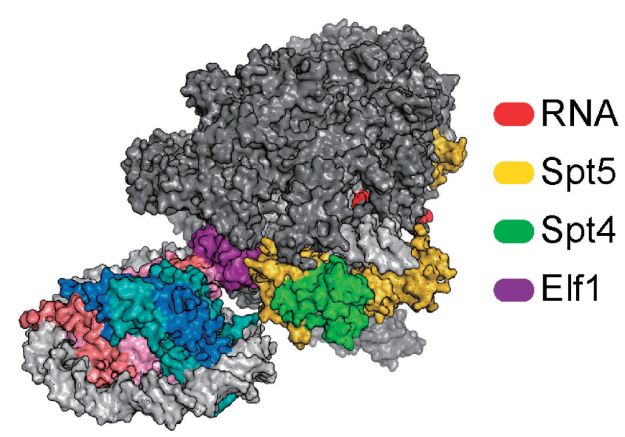

Fig. 4. Transcription mechanism through the nucleosome. A. Snapshot structures of the RNAPII-nucleosome complexes obtained using cryo-EM. RNAPII-nucleosome complexes paused at superhelical location (SHL)(-6), SHL(-5), SHL(-2), and SHL(-1) are presented. RNAPII, DNA, H2A, H2B, H3.3, and H4 are colored dark gray, gray, marine, turquoise, salmon pink, and pink, respectively. Arrows represent transitions between the complexes. B. Cryo-EM structure of the nucleosome transcribing RNAPII elongation complexes containing Elf1 and Spt4/5. The transcription elongation factors Elf1, Spt4, Spt5, and RNA are colored purple, green, yellow, and red.

the elongation factors help RNAPII to overcome the transcriptional barrier constituted by the nucleosome had remained obscure. We focused on the conserved basal elongation factors, Elf1 and Spt4/5, and analyzed them by the same method as described above. We successfully obtained cryo-EM structures of the nucleosomes with transcribing RNAPII elongation complexes containing Elf1 and $\mathrm{Spt} 4 / 5^{70)}$ (Fig. 4B). In these structures, Elf1 and Spt4/5 cooperatively facilitate RNAPII passage by modifying the interaction between RNAPII and the nucleosomal DNA, and by adjusting the nucleosome in favor of forward progression. We also found that the presence of Elf1 and Spt4/5 suppresses the pausing near the entry region of the nucleosome, by destabilizing the RNAPII-nucleosomal DNA interaction.

These structural and biochemical studies have illustrated the transcription mechanism of the nucleosomal DNA by RNAPII, bringing important insights into the basis of the transcription elongation reaction on chromatin. Further development and improvement of our structural and biochemical methods will allow us to expand these pioneering discoveries to higher-ordered structural units of chromatin, such as the chromatosome (a nucleosome complexed with the linker histone H1), OLDN, and heterochromatin units, shedding light on the complex chromatin structure-based regulatory mechanisms of eukaryotic genomes.

\section{Chromatin-related immunology and infection features}

Surprisingly, the nucleosome also functions in the regulation of the innate immune response conducted by cyclic GMP-AMP synthase (cGAS). ${ }^{71), 72)}$ cGAS senses an exogenous DNA, synthesizes a second messenger, cyclic GMP-AMP (cGAMP), and stimulates innate immunity. ${ }^{73)-76)}$ The cGAMP synthetic activity of cGAS is substantially suppressed upon nucleosome binding. ${ }^{77)} \mathrm{We}$ and others determined the cGAS-nucleosome complex structures, which revealed how cGAS is inactivated by nucleosome binding ${ }^{78)-83)}$ (Fig. 5A). Briefly, 
A
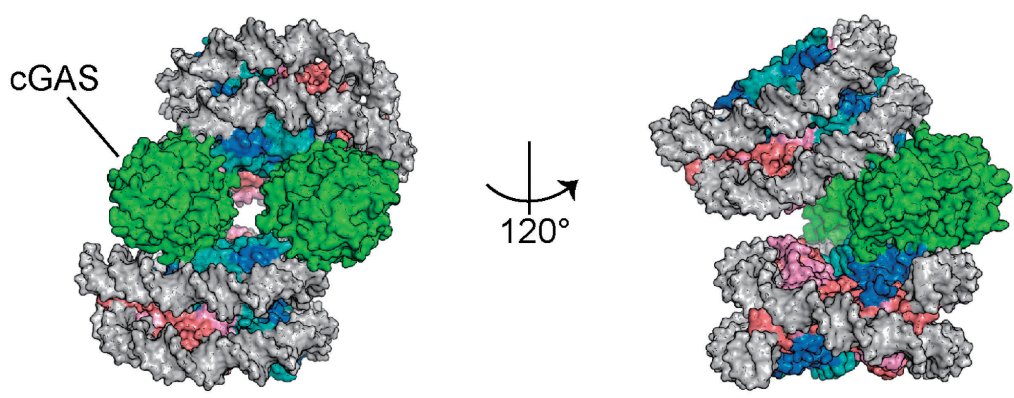

B

C
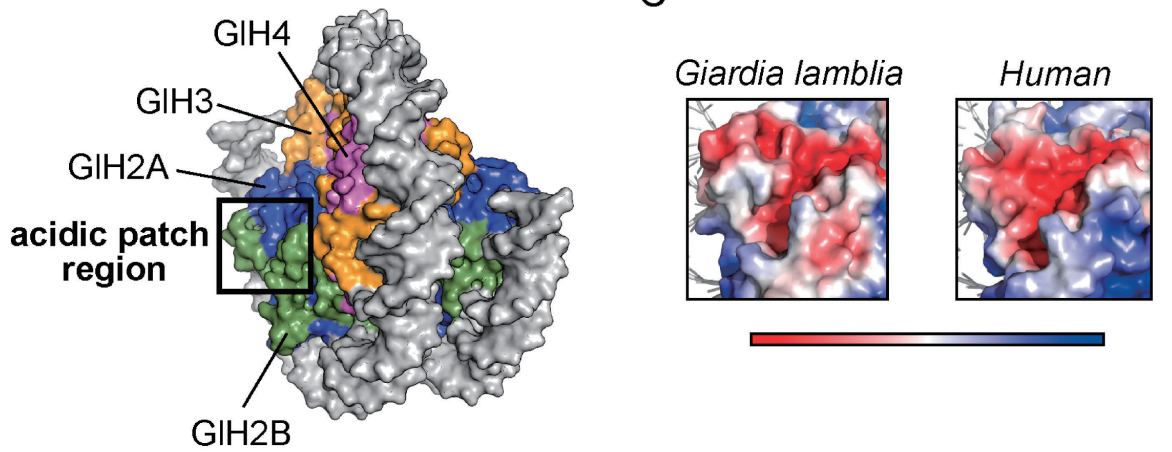

$\mathrm{D}$

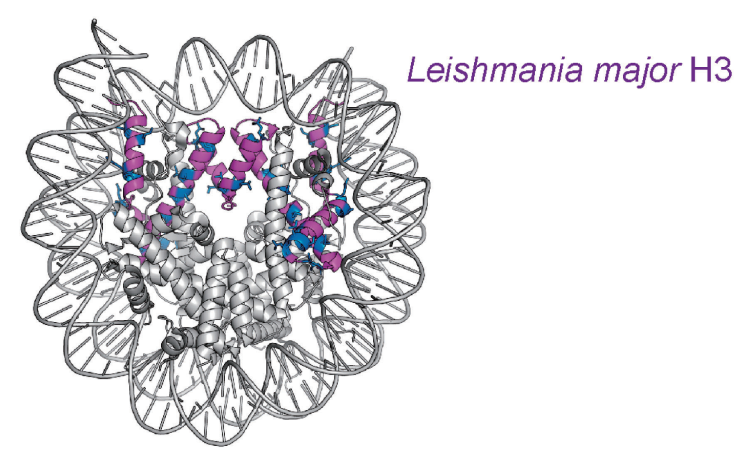

Fig. 5. Nucleosome structures implicated in immunology and in parasitic organisms. A. Cryo-EM structure of the cGAS-nucleosome complex. Two monomers of cGAS, colored green, bind the nucleosome core particle, with the four histones H2A, H2B, H3.1, and H4, colored marine, turquoise, salmon pink, and pink, respectively. The DNA is colored gray. The right panel presents the cGASnucleosome complex rotated 120 degrees counterclockwise around the axis. B. Cryo-EM structure of the Giardia lamblia nucleosome. A side view of the G. lamblia nucleosome is presented. G. lamblia histones H2A, H2B, H3, and H4 are colored blue, green, orange, and pink, respectively, with the DNA (colored gray) wrapped around the octamer. C. Close-up views around the acidic patch region in the G. lamblia nucleosome and its equivalent acidic patch region in the human nucleosome. Both panels show the electrostatic potentials of these regions, with negative and positive charges in red and blue, respectively. D. Crystal structure of the human nucleosome containing Leishmania major H3 (LmaH3). LmaH3 is colored purple, and the human H2A, H2B, H4, and DNA are colored gray. The LmaH3-specific residues are colored blue, and their side chains are depicted.

in the context of nucleosome binding, the two cGAS monomers are prevented from dimerizing, with the reorganization of each cGAS-DNA binding site in a manner incompatible with exogenous DNA binding.
This indicates that chromatin directly regulates the innate immune system. In addition, growing evidence has shown that host chromatin may act as a regulator during microbial infection. Future studies 
are awaited to define the host strategies toward infection, which may include chromatin and hostprotective elements.

We have also expanded our structural and functional studies of the nucleosome to various species, including pathogens such as Giardia lamblia, an intestinal parasite responsible for the worldwide diarrheal disease called "giardiasis", and Leishmania major, a protozoan responsible for cutaneous leishmaniasis, causing ulcerative skin lesions that heal with difficulty. The three-dimensional structure of the G. lamblia nucleosome indicated the DNA end flexibility as well as an unconventional morphology of the acidic patch region, a negatively charged region implicated in multiple chromatin interactions ${ }^{84)}$ (Fig. 5B and C). These results revealed the specific features and characteristics of the Giardia nucleosome structure and may provide important information for future drug discovery. Furthermore, inspired by the fact that Leishmania histones are secreted into human cells, we reconstituted nucleosomes consisting of human histones and Leishmania histone H3, determined the structure by X-ray crystallography, and finally proved its formation in cells ${ }^{85)}$ (Fig. 5D). This study has deepened our understanding of the mechanisms by which the host chromatin structure is altered during parasite infection.

\section{Perspective}

In over 30 years of extensive research, the structure of chromatin has been widely solved, but almost only at the first level of DNA compaction, the nucleosome. The nucleosome is an important architecture for regulating genomic DNA activity, and exhibits functional versatility in its structure and dynamics. Understanding how nucleosomes organize the higher-order chromatin states will substantially illuminate the functions and molecular mechanisms of chromatin dynamics in the epigenetic regulation of genomic DNA. With recent advances in cryo-EM technology, the elucidation of such functionally relevant higher-order chromatin structural units has become imminent. The high-resolution structures will provide important details to clarify chromatinmediated mechanisms, such as transcription. Using the cryo-EM technology, it will be quite interesting to decipher how transcription elongation is suppressed by chromatosome formation, and to determine the mechanism of transcription repression in heterochromatin. Elucidations of the functions of transcription elongation factors, histone chaperones, and remodeling factors during the chromatin transcription are also anticipated. Further developments in structural studies of chromatin, including in situ chromatin analyses, are eagerly awaited.

\section{Acknowledgements}

Thank Ms. Yukari Iikura and Ms. Yasuko Takeda (Univ. of Tokyo) for their assistance. This work was supported in part by JSPS KAKENHI grants [JP20H00449 and JP18H05534 to H.K.], grants from the Platform Project for Supporting Drug Discovery and Life Science Research (BINDS) from AMED [JP21am0101076 to H.K.], a JST CREST grant [JPMJCR16G1 to H.K.], and a JST ERATO grant [JPMJER1901 to H.K.]. I thank Mariko Dacher, Yasuko Takeda, Hiroki Tanaka, Yoshimasa Takizawa, Tomoya Kujirai, Shoko Sato, and Naoki Horikoshi for assistance in writing the manuscript.

\section{References}

1) Olins, D.E. and Olins, A.L. (2003) Chromatin history: our view from the bridge. Nat. Rev. Mol. Cell Biol. 4, 809-814.

2) Olins, A.L. and Olins, D.E. (1974) Spheroid chromatin units (v bodies). Science 183, 330-332.

3) Kornberg, R.D. (1974) Chromatin Structure: A repeating unit of histones and DNA. Science 184, 868-871.

4) Oudet, P., Gross-Bellard, M. and Chambon, P. (1975) Electron microscopic and biochemical evidence that chromatin structure is a repeating unit. Cell 4, 281-300.

5) Finch, J.T., Lutter, L.C., Rhodes, D., Brown, R.S., Rushton, B., Levitt, M. et al. (1977) Structure of nucleosome core particles of chromatin. Nature 269, 29-36.

6) Richmond, T.J., Finch, J.T., Rushton, B., Rhodes, D. and Klug, A. (1984) Structure of the nucleosome core particle at $7 \AA$ resolution. Nature 311, $532-537$.

7) Luger, K., Mäder, A.W., Richmond, R.K., Sargent, D.F. and Richmond, T.J. (1997) Crystal structure of the nucleosome core particle at $2.8 \AA$ resolution. Nature 389, 251-260.

8) Davey, C.A., Sargent, D.F., Luger, K., Maeder, A.W and Richmond, T.J. (2002) Solvent mediated interactions in the structure of the nucleosome core particle at $1.9 \AA$ resolution. J. Mol. Biol. 319, 1097-1113.

9) Richmond, T.J. and Davey, C.A. (2003) The structure of DNA in the nucleosome core. Nature 423, 145-150.

10) Wolffe, A.P. (1998) Chromatin: Structure and Function, 3rd ed. Academic Press, San Diego.

11) Freeman, L., Kurumizaka, H. and Wolffe, A.P. (1996) Functional domains for assembly of histones H3 and H4 into the chromatin of Xenopus embryos. Proc. Natl. Acad. Sci. U.S.A. 93, 12780- 
12785.

12) Kurumizaka, H. and Wolffe, A.P. (1997) Sin mutations of histone $\mathrm{H} 3$ : influence on nucleosome core structure and function. Mol. Cell. Biol. 17, 6953-6969.

13) Kruger, W. and Herskowitz, I. (1991) A negative regulator of HO transcription, SIN1 (SPT2), is a nonspecific DNA-binding protein related to HMG1. Mol. Cell. Biol. 11, 4135-4146.

14) Kruger, W., Peterson, C.L., Sil, A., Coburn, C., Arents, G., Moudrianakis, E.N. et al. (1995) Amino acid substitutions in the structured domains of histones $\mathrm{H} 3$ and $\mathrm{H} 4$ partially relieve the requirement of the yeast SWI/SNF complex for transcription. Genes Dev. 9, 2770-2779.

15) Peterson, C.L., Kruger, W. and Herskowitz, I. (1991) A functional interaction between the C-terminal domain of RNA polymerase II and the negative regulator SIN1. Cell 64, 1135-1143.

16) Wechser, M.A., Kladde, M.P., Alfieri, J.A. and Peterson, C.L. (1997) Effects of Sin-versions of histone $\mathrm{H} 4$ on yeast chromatin structure and function. EMBO J. 16, 2086-2095.

17) Muthurajan, U.M., Bao, Y., Forsberg, L.J., Edayathumangalam, R.S., Dyer, P.N., White, C.L. et al. (2004) Crystal structures of histone Sin mutant nucleosomes reveal altered proteinDNA interactions. EMBO J. 23, 260-271.

18) Cheeseman, I.M. and Desai, A. (2008) Molecular architecture of the kinetochore-microtubule interface. Nat. Rev. Mol. Cell Biol. 9, 33-46.

19) Santaguida, S. and Musacchio, A. (2009) The life and miracles of kinetochores. EMBO J. 28, 2511-2531.

20) Fukagawa, T. and Earnshaw, W.C. (2014) The centromere: chromatin foundation for the kinetochore machinery. Dev. Cell 30, 496-508.

21) McKinley, K.L. and Cheeseman, I.M. (2016) The molecular basis for centromere identity and function. Nat. Rev. Mol. Cell Biol. 17, 16-29.

22) Earnshaw, W.C. and Rothfield, N. (1985) Identification of a family of human centromere proteins using autoimmune sera from patients with scleroderma. Chromosoma 91, 313-321.

23) Cooke, C.A., Bernat, R.L. and Earnshaw, W.C. (1990) CENP-B: a major human centromere protein located beneath the kinetochore. Cell Biol. 110, 1475-1488.

24) Earnshaw, W.C., Machlin, P.S., Bordwell, B.J., Rothfield, N.F. and Cleveland, D.W. (1987) Analysis of anticentromere autoantibodies using cloned autoantigen CENP-B. Proc. Natl. Acad. Sci. U.S.A. 84, 4979-4983.

25) Masumoto, H., Masukata, H., Muro, Y., Nozaki, N. and Okazaki, T. (1989) A human centromere antigen (CENP-B) interacts with a short specific sequence in alphoid DNA, a human centromeric satellite. Cell Biol. 109, 1963-1973.

26) Muro, Y., Masumoto, H., Yoda, K., Nozaki, N., Ohashi, M. and Okazaki, T. (1992) Centromere protein $\mathrm{B}$ assembles human centromeric alphasatellite DNA at the 17-bp sequence, CENP-B box. J. Cell Biol. 116, 585-596.
27) Yoda, K., Kitagawa, K., Masumoto, H., Muro, Y. and Okazaki, T. (1992) A human centromere protein, CENP-B, has a DNA binding domain containing four potential alpha helices at the $\mathrm{NH}_{2}$ terminus, which is separable from dimerizing activity. J. Cell Biol. 119, 1413-1427.

28) Kitagawa, K., Masumoto, H., Ikeda, M. and Okazaki, T. (1995) Analysis of protein-DNA and protein-protein interactions of centromere protein B (CENP-B) and properties of the DNACENP-B complex in the cell cycle. Mol. Cell. Biol. 15, 1602-1612.

29) Tanaka, Y., Nureki, O., Kurumizaka, H., Fukai, S., Kawaguchi, S., Ikuta, M. et al. (2001) Crystal structure of the CENP-B protein-DNA complex: the DNA-binding domains of CENP-B induce kinks in the CENP-B box DNA. EMBO J. 20, 6612-6618.

30) Tawaramoto, M.S., Park, S.-Y., Tanaka, Y., Nureki, O., Kurumizaka, H. and Yokoyama, S. (2003) Crystal structure of the human centromere protein B (CENP-B) dimerization domain at $1.65-\AA$ resolution. Biol. Chem. 278, 51454-51461.

31) Tanaka, Y., Tawaramoto-Sasanuma, M., Kawaguchi, S., Ohta, T., Yoda, K., Kurumizaka, H. et al. (2004) Expression and purification of recombinant human histones. Methods 33, 3-11.

32) Kujirai, T., Arimura, Y., Fujita, R., Horikoshi, N., Machida, S. and Kurumizaka, H. (2018) Methods for preparing nucleosomes containing histone variants. Methods Mol. Biol. 1832, 3-20.

33) Tanaka, Y., Tachiwana, H., Yoda, K., Masumoto, H., Okazaki, T., Kurumizaka, H. et al. (2005) Human centromere protein B induces translational positioning of nucleosomes on alpha-satellite sequences. J. Biol. Chem. 80, 41609-41618.

34) Fujita, R., Otake, K., Arimura, Y., Horikoshi, N., Miya, Y., Shiga, T. et al. (2015) Stable complex formation of CENP-B with the CENP-A nucleosome. Nucleic Acids Res. 43, 4909-4922.

35) Black, B.E. and Cleveland, D.W. (2011) Epigenetic centromere propagation and the nature of CENPA nucleosomes. Cell 144, 471-479.

36) Tachiwana, H., Kagawa, W., Shiga, T., Osakabe, A., Miya, Y., Saito, K. et al. (2011) Crystal structure of the human centromeric nucleosome containing CENP-A. Nature 476, 232-235.

37) Tsunaka, Y., Kajimura, N., Tate, S. and Morikawa, K. (2005) Alteration of the nucleosomal DNA path in the crystal structure of a human nucleosome core particle. Nucleic Acids Res. 33, 3424-3434.

38) Tachiwana, H., Osakabe, A., Shiga, T., Miya, Y., Kimura, H., Kagawa, W. et al. (2011) Structures of human nucleosomes containing major histone H3 variants. Acta Crystallogr. D Biol. Crystallogr. 67, 578-583

39) Roulland, Y., Ouararhni, K., Naidenov, M., Ramos, L., Shuaib, M., Syed, S.H. et al. (2016) The flexible ends of CENP-A nucleosome are required for mitotic fidelity. Mol. Cell 63, 674-685.

40) Boopathi, R., Danev, R., Khoshouei, M., Kale, S., Nahata, S., Ramos, L. et al. (2020) Phase-plate 
cryo-EM structure of the Widom 601 CENP-A nucleosome core particle reveals differential flexibility of the DNA ends. Nucleic Acids Res. 48, $5735-5748$

41) Takizawa, Y., Ho, C.-H., Tachiwana, H., Matsunami, H., Kobayashi, W., Suzuki, M. et al. (2020) Cryo-EM structures of centromeric trinucleosomes containing a central CENP-A nucleosome. Structure 28, 44-53.e4.

42) Arimura, Y., Tachiwana, H., Takagi, H., Hori, T., Kimura, H., Fukagawa, T. et al. (2019) The CENP-A centromere targeting domain facilitates H4K20 monomethylation in the nucleosome by structural polymorphism. Nat. Commun. 10, 576.

43) Ho, C.-H., Takizawa, Y., Kobayashi, W., Arimura, Y., Kimura, H. and Kurumizaka, H. (2021) Structural basis of nucleosomal histone H4 lysine 20 methylation by SET8 methyltransferase. Life Sci. Alliance 4, e202000919.

44) Hori, T., Shang, W.H., Toyoda, A., Misu, S., Monma, N., Ikeo, K. et al. (2014) Histone H4 Lys 20 monomethylation of the CENP-A nucleosome is essential for kinetochore assembly. Dev. Cell 29, 740-749.

45) Lacoste, N., Woolfe, A., Tachiwana, H., Garea, A.V., Barth, T., Cantaloube, S. et al. (2014) Mislocalization of the centromeric histone variant CenH3/ CENP-A in human cells depends on the chaperone DAXX. Mol. Cell 53, 631-644.

46) Arimura, Y., Shirayama, K., Horikoshi, N., Fujita, R., Taguchi, H., Kagawa, W. et al. (2014) Crystal structure and stable property of the cancerassociated heterotypic nucleosome containing CENP-A and H3.3. Sci. Rep. 4, 7115.

47) Tachiwana, H., Osakabe, A., Kimura, H. and Kurumizaka, H. (2008) Nucleosome formation with the testis-specific histone $\mathrm{H} 3$ variant, H3t, by human nucleosome assembly proteins in vitro. Nucleic Acids Res. 36, 2208-2218.

48) Tachiwana, H., Kagawa, W., Osakabe, A., Kawaguchi, K., Shiga, T., Hayashi-Takanaka, Y. et al. (2010) Structural basis of instability of the nucleosome containing a testis-specific histone variant, human H3T. Proc. Natl. Acad. Sci. U.S.A. 107, 10454-10459.

49) Maehara, K., Harada, A., Sato, Y., Matsumoto, M., Nakayama, K.-I., Kimura, H. et al. (2015) Tissuespecific expression of histone $\mathrm{H} 3$ variants diversified after species separation. Epigen. Chrom. 17, $8-35$.

50) Ueda, J., Harada, A., Urahama, T., Machida, S., Maehara, K., Hada, M. et al. (2017) Testis-specific histone variant H3t gene is essential for entry into spermatogenesis. Cell Rep. 18, 593-600.

51) Urahama, T., Harada, A., Maehara, K., Horikoshi, N., Sato, K., Sato, Y. et al. (2016) Histone H3.5 forms an unstable nucleosome and accumulates around transcription start sites in human testis. Epigen. Chrom. 9, 2.

52) Urahama, T., Horikoshi, N., Osakabe, A., Tachiwana, H. and Kurumizaka, H. (2014) Structure of human nucleosome containing the testis- specific histone variant TSH2B. Acta Crystallogr. F Struct. Biol. Commun. 70, 444-449.

53) Montellier, E., Boussouar, F., Rousseaux, S., Zhang, K., Buchou, T., Fenaille, F. et al. (2013) Chromatin-to-nucleoprotamine transition is controlled by the histone H2B variant TH2B. Genes Dev. 27, 1680-1692.

54) Shinagawa, T., Huynh, L.M., Takagi, T., Tsukamoto, D., Tomaru, C., Kwak, H.G. et al. (2015) Disruption of Th2a and Th2b genes causes defects in spermatogenesis. Development 142, 1287-1292.

55) Padavattan, S., Shinagawa, T., Hasegawa, K., Kumasaka, T., Ishii, S. and Kumarevel, T. (2015) Structural and functional analyses of nucleosome complexes with mouse histone variants TH2a and TH2b, involved in reprogramming. Biochem. Biophys. Res. Commun. 464, 929-935.

56) Padavattan, S., Thiruselvam, V., Shinagawa, T., Hasegawa, K., Kumasaka, T., Ishii, S. et al. (2017) Structural analyses of the nucleosome complexes with human testis-specific histone variants, hTh2a and hTh2b. Biophys. Chem. 221, 41-48.

57) Barral, S., Morozumi, Y., Tanaka, H., Montellier, E., Govin, J., de Dieuleveult, M. et al. (2017) Histone variant H2A.L.2 guides transition protein-dependent protamine assembly in male germ cells. Mol. Cell 66, 89-101.e8.

58) Machida, S., Takizawa, Y., Ishimaru, M., Sugita, Y., Sekine, S., Nakayama, J.-I. et al. (2018) Structural basis of heterochromatin formation by human HP1. Mol. Cell 69, 385-397.e8.

59) Poepsel, S., Kasinath, V. and Nogales, E. (2018) Cryo-EM structures of PRC2 simultaneously engaged with two functionally distinct nucleosomes. Nat. Struct. Mol. Biol. 25, 154-162.

60) Zaret, K.S. and Carroll, J.S. (2011) Pioneer transcription factors: establishing competence for gene expression. Rev. Genes Dev. 25, 2227-2241.

61) Takaku, M., Grimm, S.A., Shimbo, T., Perera, L., Menafra, R., Stunnenberg, H.G. et al. (2016) GATA3-dependent cellular reprogramming requires activation-domain dependent recruitment of a chromatin remodeler. Genome Biol. 27, 17-36.

62) Tanaka, H., Takizawa, Y., Takaku, M., Kato, D., Kumagawa, Y., Grimm, S.A. et al. (2020) Interaction of the pioneer transcription factor GATA3 with nucleosomes. Nat. Commun. 11, 4136.

63) Michael, A.K., Grand, R.S., Isbel, L., Cavadini, S., Kozicka, Z., Kempf, G. et al. (2020) Mechanisms of OCT4-SOX2 motif readout on nucleosomes. Science 368, 1460-1465.

64) Dodonova, S.O., Zhu, F., Dienemann, C., Taipale, J. and Cramer, P. (2020) Nucleosome-bound SOX2 and SOX11 structures elucidate pioneer factor function. Nature 580, 669-672.

65) Ulyanova, N.P. and Schnitzler, G.R. (2005) Human SWI/SNF generates abundant, structurally altered dinucleosomes on polynucleosomal templates. Mol. Cell. Biol. 25, 11156-11170.

66) Engeholm, M., de Jager, M., Flaus, A., Brenk, R., van Noort, J. and Owen-Hughes, T. (2009) 
Nucleosomes can invade DNA territories occupied by their neighbors. Nat. Struct. Mol. Biol. 16, 151158.

67) Kato, D., Osakabe, A., Arimura, Y., Mizukami, Y., Horikoshi, N., Saikusa, K. et al. (2017) Crystal structure of the overlapping dinucleosome composed of hexasome and octasome. Science $\mathbf{3 5 6}$ 205-208.

68) Matsumoto, A., Sugiyama, M., Li, Z., Martel, A., Porcar, L., Inoue, R. et al. (2020) Structural studies of overlapping dinucleosomes in solution. Biophys. J. 118, 2209-2219.

69) Kujirai, T., Ehara, H., Fujino, Y., Shirouzu, M., Sekine, S.-I. and Kurumizaka, H. (2018) Structural basis of the nucleosome transition during RNA polymerase II passage. Science 362, 595-598.

70) Ehara, H., Kujirai, T., Fujino, Y., Shirouzu, M. Kurumizaka, H. and Sekine, S.-I. (2019) Structural insight into nucleosome transcription by RNA polymerase II with elongation factors. Science 363, 744-747.

71) Ablasser, A. and Chen, Z.J. (2019) cGAS in action: Expanding roles in immunity and inflammation. Science 363, 6431.

72) Motwani, M., Pesiridis, S. and Fitzgerald, K.A. (2019) DNA sensing by the cGAS-STING pathway in health and disease. Nat. Rev. Genet. 20, 657674.

73) Li, X., Shu, C., Yi, G., Chaton, C.T., Shelton, C.L., Diao, J. et al. (2013) Cyclic GMP-AMP synthase is activated by double-stranded DNA-induced oligomerization. Immunity 39, 1019-1031.

74) Gao, P., Ascano, M., Wu, Y., Barchet, W., Gaffney, B.L., Zillinger, T. et al. (2013) Cyclic $\left[\mathrm{G}\left(2^{\prime}, 5^{\prime}\right) \mathrm{pA}\left(3^{\prime}, 5^{\prime}\right) \mathrm{p}\right]$ is the metazoan second messenger produced by DNA-activated cyclic GMPAMP synthase. Cell 153, 1094-1107.

75) Hooy, R.M. and Sohn, J. (2018) The allosteric activation of cGAS underpins its dynamic signaling landscape. eLife 7, e39984.

76) Xie, W., Lama, L., Adura, C., Tomita, D., Glickman, J.F., Tuschl, T. et al. (2019) Human cGAS catalytic domain has an additional DNA-binding interface that enhances enzymatic activity and liquid-phase condensation. Proc. Natl. Acad. Sci. U.S.A. 116, 11946-11955.

77) Zierhut, C., Yamaguchi, N., Paredes, M., Luo, J.D., Carroll, T. and Funabiki, H. (2019) The cytoplasmic DNA sensor cGAS promotes mitotic cell death. Cell 178, 302-315.e23.

78) Kujirai, T., Zierhut, C., Takizawa, Y., Kim, R., Negishi, L., Uruma, N. et al. (2020) Structural basis for the inhibition of cGAS by nucleosomes. Science 370, 455-458.

79) Boyer, J.A., Spangler, C.J., Strauss, J.D., Cesmat, A.P., Liu, P., McGinty, R.K. et al. (2020) Structural basis of nucleosome-dependent cGAS inhibition. Science 370, 450-454.

80) Pathare, G.R., Decout, A., Glück, S., Cavadini, S., Makasheva, K., Hovius, R. et al. (2020) Structural mechanism of cGAS inhibition by the nucleosome. Nature 587, 668-672.

81) Michalski, S., de Oliveira Mann, C.C., Stafford, C.A., Witte, G., Bartho, J., Lammens, K. et al. (2020) Structural basis for sequestration and autoinhibition of cGAS by chromatin. Nature 587, 678-682.

82) Zhao, B., Xu, P., Rowlett, C.M., Jing, T., Shinde, O., Lei, Y. et al. (2020) The molecular basis of tight nuclear tethering and inactivation of cGAS. Nature 587, 673-677.

83) Cao, D., Han, X., Fan, X., Xu, R.M. and Zhang, X. (2020) Structural basis for nucleosome-mediated inhibition of cGAS activity. Cell Res. 30, 10881097.

84) Sato, S., Takizawa, Y., Hoshikawa, F., Dacher, M., Tanaka, H., Tachiwana, H. et al. (2021) Cryo-EM structure of the nucleosome core particle containing Giardia lamblia histones. Nucleic Acids Res. 49, 8934-8946.

85) Dacher, M., Tachiwana, H., Horikoshi, N., Kujirai, T., Taguchi, H., Kimura, H. et al. (2019) Incorporation and influence of Leishmania histone $\mathrm{H} 3$ in chromatin. Nucleic Acids Res. 47, 11637-11648.

(Received Sep. 29, 2021; accepted Nov. 10, 2021) 


\section{Profile}

Hitoshi Kurumizaka was born in Nagoya in 1967. He graduated from the Graduate School of Pharmaceutical Science at the Tokyo College of Pharmacy in 1991. He obtained his Ph.D. degree from Saitama University in 1995 focusing on mechanisms of DNA recombination. He joined the National Institutes of Health in the U.S.A. as a postdoctoral fellow, where he broadened his scientific interest to the chromatin field. In 1997, he moved to RIKEN institute as a research scientist, and then in 2003 to the Graduate School of Science and Engineering, Waseda University, as an independent associate professor, and became a full professor in 2008. He has developed various in vitro chromatin reconstitution methods and studied many nucleosomes and chromatin units by unraveling their structural and biochemical properties. In 2018, he moved to Institute

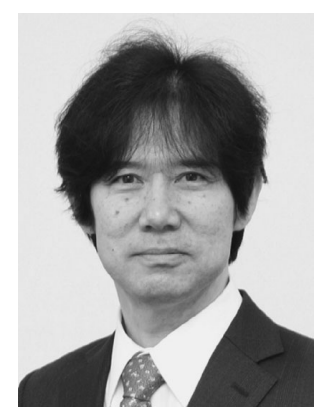
for Quantitative Biosciences, the University of Tokyo, as a full professor. His ongoing research interests are focused on chromatin structure-based, epigenetic regulatory mechanisms of genomic DNA in eukaryotes. For his accomplishments, he received awards such as the Award for Science and Technology (Research Category), the Commendation for Science and Technology by the Minister of Education, Culture, Sports, Science and Technology (2021), Academic Award of the Mochida Memorial Foundation (2020), Samuro Kakiuchi Memorial Award by Gushinkai (The Japanese Biochemical Society) (2018). He serves as a standing director of The Japanese Biochemical Society, an operating officer of the Protein Science Society of Japan, and a committee member of The Japanese Society for Epigenetics. He is a member of the Editorial Board of the EMBO Journal. 